\title{
Antimicrobial resistance in food
}

\author{
Scott A McEwen DVM DVSc Diplomate ACVP ${ }^{1}$, Richard Reid-Smith DVM DVSc ${ }^{1,2}$
}

$\mathrm{N}$ ewspapers and other media provide almost daily reminders to Canadians of the potential risks of foodborne infection. The recent discovery of a case of bovine spongiform encephalopathy in this country and its dramatic impact on farming and international trade show some of the indirect effects of foodborne contaminants on society. But it's more than media hype; our provincial and national notifiable disease surveillance programs provide abundant evidence of the direct effects on morbidity and mortality from a wide range of food and waterborne contaminants, such as Salmonella, Campylobacter, Escherichia coli O157:H7 and other bacterial (eg, Listeria, Yersinia), parasitic (eg, Cyclospora, Toxoplasma) and viral (eg, norovirus) infections.

What about antimicrobial resistance as a food safety issue? Understandably, methicillin-resistant Staphylococcus aureus, vancomycin-resistant enterococci, and resistance among respiratory, genitourinary and some other human-derived bacterial infections receive top billing on lists of resistance problems facing the medical community (1). For these infections, antimicrobial prescribing practices in hospitals and the community can often be directly linked to emergence of resistance. Not to be forgotten, however, is resistance among food- and waterborne bacteria, where resistance also increases the burden of illness, but where animals and other environmental sources are major reservoirs of infection. Therefore, in contrast to the major nosocomial and community-acquired bacterial infections that have predominantly human reservoirs, we look to nonhuman use of antimicrobials as potential drivers of resistance in many of the food- and waterborne bacteria of animal origin (eg, the zoonoses). Most of the nonhuman use of antimicrobials is in food animals, although they are also used in pets, horses and in some types of plant production (eg, prophylaxis of some bacterial infections of fruit), but these are thought to be of lesser importance, at least to the extent that they have been studied (2).

The article by Forward and colleagues (pages 226-230) in this issue of the Journal provides an excellent example of the potential for food to be a vehicle of infection because of antimicrobial-resistant pathogens and commensals. It also raises a number of issues that are central to the ongoing and often highly contentious discussion of nonhuman use of antimicrobials and whether changes in current practice are needed. These issues include the use in animals of antimicrobial drugs of critical importance in human medicine, the challenges to understanding and controlling resistance that are posed by the complexity of the food chain and the general ecology of resistance, the need for surveillance of both resistance and antimicrobial use, and the importance of improved food safety activities throughout the farm-to-fork continuum, including prudent use of antimicrobials in animals.

Many foodborne bacteria are quite capable of causing illness in humans in the absence of antimicrobial resistance (eg, E coli O157:H7 and many Salmonella and Campylobacter infections), however, there are a number of mechanisms by which antimicrobial resistance in foodborne bacteria can increase the burden of illness. These mechanisms include: rendering infections more difficult or expensive to treat; enhancing virulence or pathogenicity, resulting in more severe or longer-lasting disease; increasing risk of infection (in particular among resistant Salmonella) in people taking antimicrobials for other reasons through reduction of colonization resistance; contributing to the pool of resistance determinants available for uptake by other human pathogens; and enhancing the spread of zoonotic infections in animals undergoing antimicrobial therapy, making these infections more available for human infection by direct or indirect means (3).

The prospect of treatment failure, especially in lifethreatening situations, is perhaps the most intuitively obvious and serious of these impacts. However, an expert panel assessing salmonellosis risks from the subtherapeutic use of penicillin and tetracycline in animal feed considered that the greatest public health impact from resistance in Salmonella was probably the so-called 'etiologic fraction', those cases of salmonellosis that occurred because the infections were resistant (eg, associated with reduced colonization resistance from prior antimicrobial therapy), and the resistance was attributable to the use of antimicrobials in animals (4).

Not surprisingly, the importance of antimicrobials to human health is one of the criteria being used to assess risks of nonhuman uses of antimicrobials, along with considerations of the organisms involved, methods of antimicrobial treatment in animals and other factors $(5,6)$. Classification of a drug or class as critically important for human health may include consideration of its importance in treatment of enteric infections in humans, whether it is the only available therapy or one of few alternatives for treating serious human disease, and whether there is cross-resistance with other highly important drugs or known linked resistance to other important classes. Examples include third-generation cephalosporins, fluoroquinolones and glycopeptides, among others.

Physicians and veterinarians are accustomed to assessing risks to health using direct evidence that may be acquired from

${ }^{1}$ Department of Population Medicine, Ontario Veterinary College, University of Guelph; ${ }^{2}$ Laboratory for Foodborne Zoonoses, Population and Public Health Branch, Health Canada, Guelph, Ontario

Correspondence and reprints: Dr Scott McEwen, Department of Population Medicine, Ontario Veterinary College, University of Guelph, Guelph, Ontario N1G 2W1. Telephone 519-824-4120 ext 54751, fax 519-763-3117, e-mail smcewen@uoguelph.ca 
clinical experience, or, preferably, through controlled studies that are either observational or experimental in design. Unfortunately, public health risks from nonhuman uses of antimicrobials do not easily lend themselves to this direct approach, largely for logistical reasons because it is a long way from the farm to the patient, not only in geographical terms, but also in other regards. A multitude of variables can affect the dynamics of microbial infections in animals, food and the environment, and these can operate at the levels of food production, processing, distribution (local, regional, international), food preparation and foodservice industries. Even the ambitious studies that span the farm-to-fork continuum have difficulties incorporating the 'legacy' effect of resistance, the notion that antimicrobial use at one time and place may affect resistance that is observed far away, in other species of animals, perhaps months or years later. Given these formidable barriers to comprehensive assessment of risk through direct study, we are now learning from the experiences in environmental health and engineering, and we are increasingly adopting indirect methods of risk assessment. An important aspect of these risk assessments is to provide some appreciation of the magnitude of public health impact; for example, the number of people affected and the severity of illness. Published examples include the previously mentioned Institute of Medicine assessment of risk from subtherapeutic use of penicillin and tetracycline in animal feed, which focused on Salmonella-related mortality (4), the US Food and Drug Administration assessment of risk from fluoroquinolone use in poultry, which focused on Campylobacter-related morbidity (7), and an assessment of risks from macrolide use in animals, where the adverse effects of interest were human illnesses caused by macrolide-resistant Campylobacter or macrolide-resistant Enterococcus faecium (8).

A better understanding of human health risks is essential for the development of voluntary and regulatory programs that support prudent nonhuman use of antimicrobials. There are some resistance problems in the major pathogens of animals (usually strains of bacteria which are not of importance to human health), but the supply of new antimicrobial drugs is not yet exhausted in veterinary medicine, although many believe that point may soon come (9), and farmers and veterinarians do not yet perceive that there is a resistance crisis in animal health, as there is in human health. Perhaps this perception will change, but for the time being, the major motive for controlling resistance problems in animals is to protect human health. However, many farmers and veterinarians are not yet convinced that their practices significantly endanger human health, or that such risks that do exist warrant actions on their part, especially drastic ones (eg, banning certain classes or types of antimicrobial use) that they believe will increase their cost of production, make them uncompetitive in national or international markets, or leave them without medication to treat their animals when necessary. Fairly or unfairly, farmers and veterinarians sometimes feel they are being blamed for the majority of resistance problems in humans. Clearly, better communication and education programs are needed to improve understanding of the efforts underway in the human health sector to control resistance, and vice versa.

Fortunately, research in Canada and abroad is beginning to improve understanding of resistance in animals, the effects of changes in antimicrobial use practices, and alternatives to antimicrobials. Furthermore, it is clear that the great majority of farmers and veterinarians will take appropriate action when needed, and the agricultural community in Canada has a strong history of dealing with important zoonoses, such as brucellosis and bovine tuberculosis. Food safety programs are emerging throughout the food chain as all players in the diverse food industry grasp the importance of food safety, and as techniques such as Hazard Analysis, Critical Control Point and on-farm quality assurance programs are developed to achieve pathogen reduction goals. The current challenge is to incorporate antimicrobial resistance and prudent use considerations into these programs.

Forward and colleagues draw attention to the need for longitudinal studies of resistance in food to better understand temporal trends, and follow spread of resistance elements and the bacteria that harbour them. They also point out the lack of specific information on antimicrobial use in animals, which hampers understanding of the role of such use in resistance selection. These are powerful arguments for surveillance of antimicrobial resistance in animals and food, and surveillance of nonhuman antimicrobial use. Unfortunately, the lack of surveillance in these areas has impeded research and control efforts for decades, but there has been marked improvement in recent years. For example, much of what we know of antimicrobial resistance in Salmonella in animals, food, the environment and humans is because of integrated human-food-animal surveillance in the United Kingdom (10), although, until recently, British authorities were not able to link these data to antimicrobial use information at the regional or national level. Similarly, the United States has its National Antimicrobial Resistance Monitoring System, which includes human, animal, and food isolates of Salmonella and other species of bacteria (11), but it also does not currently link to antimicrobial use data because a mechanism for its collection does not yet exist. Denmark leads the way internationally in integrated surveillance of resistance and antimicrobial use in both animals and humans, through its Danish Integrated Antimicrobial Resistance Monitoring Programme (DANMAP) for surveillance of resistance in foodborne pathogens and commensals, and the Veterinary Medicine Statistics (VETSTAT) program for surveillance of antimicrobial use (12). The Danish programs have provided crucial information on the relationships between various types of antimicrobial use in animals and resistance in animals, food and humans, and on the effects of changes in antimicrobial use policies (eg, banning of antimicrobial growth promoters) on resistance, food safety, animal production, and animal and human health.

Improved surveillance of resistance and antimicrobial use in Canada were among the recommendations made by recent national expert panels $(13,14)$. A major step forward was the development of the Canadian Integrated Program for Antimicrobial Resistance Surveillance (CIPARS), which released its first report of 2002 data in March of 2004 (6). CIPARS seeks to monitor trends in antimicrobial use and resistance in a variety of bacteria (eg, Salmonella, Campylobacter, E coli, enterococci) from human, animal and food sources across Canada. An important early goal was to test the feasibility of an epidemiologically representative surveillance system using sampling and microbiological methods that are unified and comparable with those employed in other jurisdictions. Although somewhat limited in its first incarnation, being primarily focused on data derived from active 
surveillance in slaughter plants, passive surveillance of Salmonella and human antimicrobial use monitoring, the CIPARS report will be expanded in the near future to include data derived from active surveillance of antimicrobial resistance in retail meat, enhanced surveillance of antimicrobial resistance in human Salmonella, on-farm surveillance of antimicrobial resistance and monitoring of antimicrobial use in animals. Importantly, CIPARS antimicrobial resistance and use monitoring data will be used for human health risk assessment and to support science-based policies for control of antimicrobial resistance, including resistance arising from nonhuman use of antimicrobials. The 2002 CIPARS report provides valuable national data on antimicrobial resistance in the food chain, and it is expected that future CIPARS reports will enable more comprehensive analysis of trends and associations

\section{REFERENCES}

1. Department of Health, UK. The path of least resistance. Main report of the Standing Medical Advisory Committee, sub-group on antimicrobial resistance. London: Department of Health, 1998:33-46.

2. Vidaver A. Uses of antimicrobials in plant agriculture. Clin Infect Dis 2002;34(Suppl 3):S107-10.

3. Barza M. Potential mechanisms of increased disease in humans from antimicrobial resistance in food animals. Clin Infect Dis 2002;34(Suppl 3):S123-5.

4. Institute of Medicine. Human Health Rrisks with the Subtherapeutic use of Penicillin or Tetracyclines in Animal Feed. Washington: National Academy Press, 1989.

5. Food and Drug Administration, Center for Veterinary Medicine. Guidance for industry \#152: Evaluating the safety of antimicrobial new animal drugs with regard to their microbiological effects on bacteria of human health concern. Food and Drug Administration, 2003. <http://www.fda.gov/cvm/antimicrobial/antimicrobial.html> (Version current at August 4, 2004).

6. Health Canada. Canadian Integrated Program for Antimicrobial Resistance Surveillance (CIPARS) 2002. Health Canada 2004. <http://www.hc-sc.gc.ca/pphb-dgspsp/cipars-picra/index.html> (Version current at August 4, 2004).

7. Food and Drug Administration, Center for Veterinary Medicine. Risk assessment on the human health impact of fluoroquinoloneresistant Campylobacter associated with the consumption of chicken. Food and Drug Administration, 2001.

<http://www.fda.gov/cvm/antimicrobial/Risk_asses.htm> (Version current at August 4, 2004).

8. Hurd HS, Doores S, Hayes D, et al. Public health consequences of macrolide use in food animals: A deterministic risk assessment. J Food Prot 2004;67:980-92. between nonhuman antimicrobial use and resistance in animals, food and humans.

While essential, national surveillance programs alone are not sufficient for providing us the answers we need in some key areas, including the emergence, dissemination, persistence and decline of antimicrobial resistance in the food chain, including multiresistant clones of Salmonella, the association between resistance and virulence, the importance of nonantimicrobial risk factors in the spread of resistance, and the role of commensals as reservoirs of resistance. These and other issues are being addressed in targeted studies at many research centres in Canada and around the world. The next five years should witness a quantum leap forward in our understanding of the dynamics of antimicrobial resistance in the food chain, its effects on human health and optimal methods of control.

9. Reid-Smith RJ, Imgrund R, Deckert A, Dewey C, Richardson K, McEwen SA. Attitudes towards antimicrobial resistance and antimicrobial drug use patterns of Canadian swine veterinarians. In: Proceedings of the 2nd International Conference on Antimicrobial Agents in Veterinary Medicine; June 13-17, 2004, Ottawa, Canada.

10. Threlfall EJ, Teale CJ, Davies RH, et al. A comparison of antimicrobial susceptibilities in nontyphoidal salmonellas from humans and food animals in England and Wales in 2000. Microb Drug Resist 2003;9:183-9.

11. Centers for Disease Control and Prevention. National Antimicrobial Resistance Monitoring System (NARMS) for enteric bacteria (1996-1999). Atlanta: Centers for Disease Control and Prevention, 1999.

12. World Health Organization. International Review Panel Evaluation of the Termination of the Use of Antimicrobial Growth Promoters in Denmark. World Health Organization, 2003 $<$ http://www.who.int/salmsurv/links/gssamrgrowthreportstory/en/> (Version current at August 4, 2004).

13. Anonymous. Uses of antimicrobials in food animals in Canada: Impact on resistance and human health. Report of Health Canada's Advisory Committee on animal uses of antimicrobial and impact on resistance and human health. Ottawa: Health Canada, 2002. <http://www.hc-sc.gc.ca/vetdrugs-medsvet/index_e.html> (Version current at August 4, 2004).

14. Health Canada and the Infectious Disease Society. Controlling antimicrobial resistance: An integrated action plan for Canadians. Canadian communicable disease report 1997;23S7. <http://www.hcsc.gc.ca/hpb/lcdc/publicat/ccdr/97vol23/vol23s7/index.html> (Version current at August 4, 2004). 


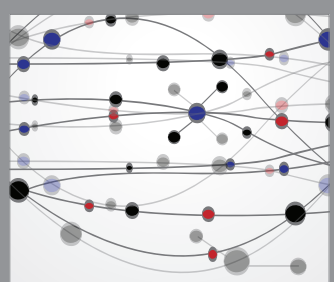

The Scientific World Journal
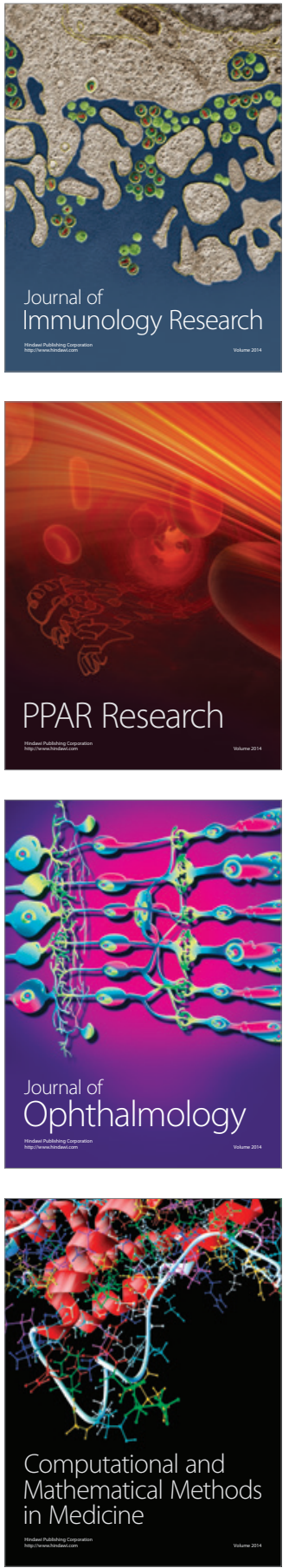

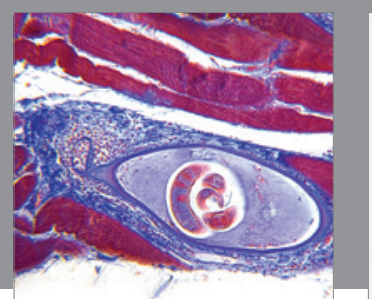

Gastroenterology Research and Practice

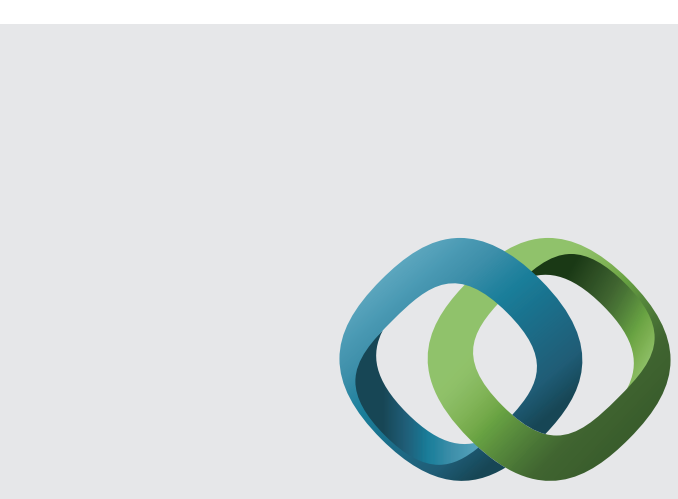

\section{Hindawi}

Submit your manuscripts at

http://www.hindawi.com
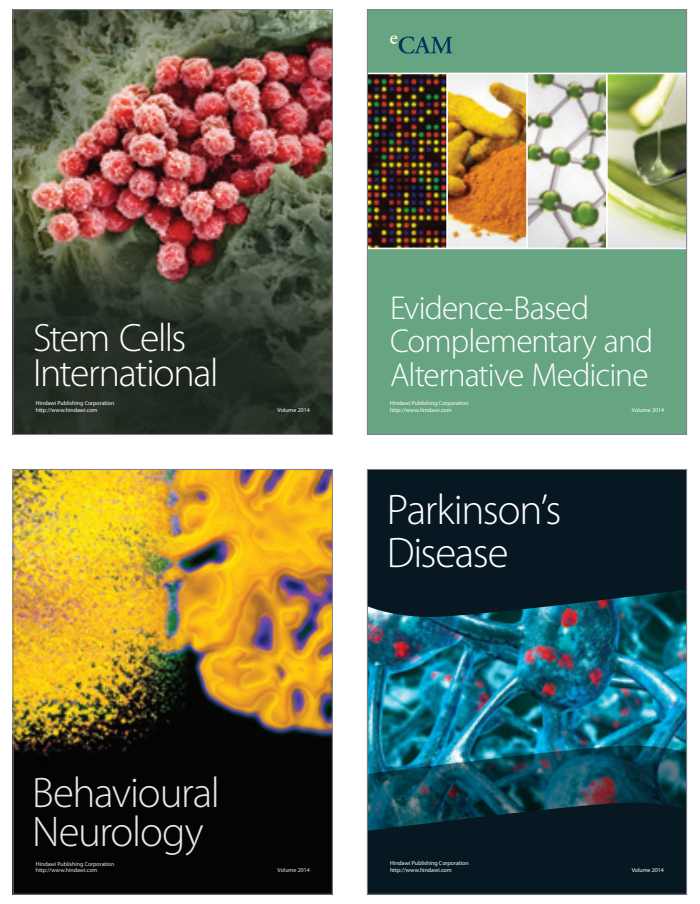
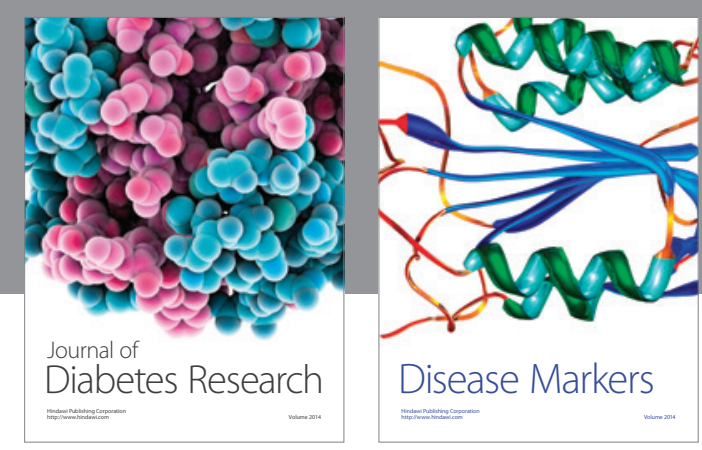

Disease Markers
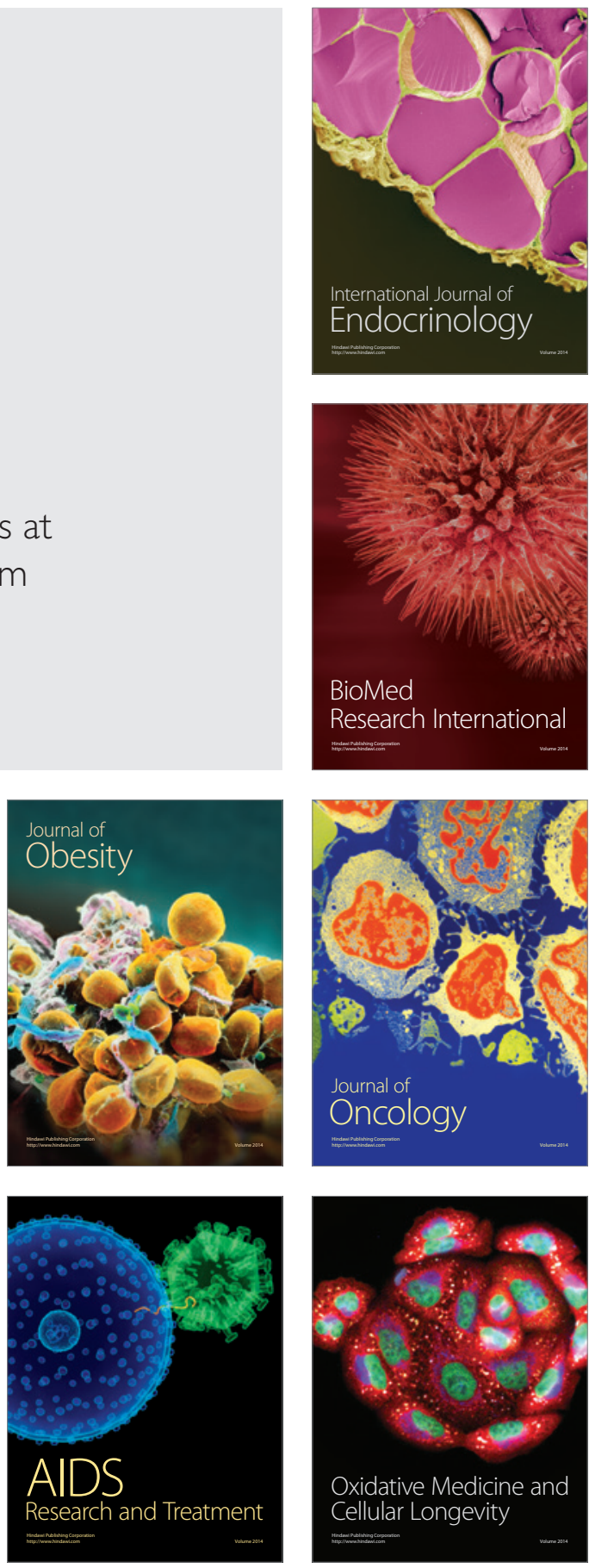